Bryan D. Riggeal, MD

Beau B. Bruce, MD, MS

Amit M. Saindane, MD

Maysa A. Ridha, MD

Linda P. Kelly, MD

Nancy J. Newman, MD

Valérie Biousse, MD

Correspondence to

Dr. Biousse:

vbiouss@emory.edu

\section{Clinical course of idiopathic intracranial hypertension with transverse sinus stenosis}

[1]

Objective: Transverse sinus stenosis (TSS) is common in idiopathic intracranial hypertension (IIH), but its effect on the course and outcome of IIH is unknown. We evaluated differences in TSS characteristics between patients with IIH with "good" vs "poor" clinical courses.

Methods: All patients with IIH seen in our institution after September 2009 who underwent a high-quality standardized brain magnetic resonance venogram (MRV) were included. Patients were categorized as having a good or poor clinical course based on medical record review. The location and percent of each TSS were determined for each patient, and were correlated to the clinical outcome.

Results: We included 51 patients. Forty-six patients had bilateral TSS. The median average percent stenosis was $56 \%$. Seventy-one percent of patients had stenoses $>50 \%$. Thirty-five of the 51 patients (69\%) had no final visual field loss. Eight patients (16\%) had a clinical course classified as poor. There was no difference in the average percent stenosis between those with good clinical courses vs those with poor courses $(62 \%$ vs $56 \%, p=0.44)$. There was no difference in the percent stenosis based on the visual field grade $(p=0.38)$. CSF opening pressure was not associated with either location or degree of TSS.

Conclusion: TSS is common, if not universal, among patients with $\mathrm{IH}$, and is almost always bilateral. There is no correlation between the degree of TSS and the clinical course, including visual field loss, among patients with $\mathrm{IH}$, suggesting that clinical features, not the degree of TSS, should be used to determine management in IIH. Neurology ${ }^{\circledR} 2013 ; 80: 289-295$

\title{
GLOSSARY
}

ATECO = auto-triggered elliptic-centric-ordered; GRE = gradient-recalled echo; GVF = Goldmann visual field; HVF = Humphrey visual field; ICP = intracranial pressure; IIH = idiopathic intracranial hypertension; IQR = interquartile range; $\mathbf{L P}=$ lumbar puncture; MIP = maximum intensity projections; $\mathbf{M R V}=$ magnetic resonance venography; $\mathbf{T S}=$ transverse sinus; $\mathbf{T S S}=$ transverse sinus stenosis; $\mathbf{V F}=$ visual field

Idiopathic intracranial hypertension (IIH) is a syndrome of isolated elevated intracranial pressure (ICP) of unknown etiology, responsible for visual loss from papilledema in about $25 \%$ of cases. ${ }^{1}$ Transverse sinus stenosis (TSS) is commonly described in patients with IIH, and likely contributes to intracranial hypertension via venous hypertension when the stenosis is bilateral and severe enough to compromise cerebral venous outflow. ${ }^{2-4}$ Previous reports $^{5-7}$ have described improvement in these stenoses after CSF removal, suggesting that some stenoses may be secondary to increased ICP rather than causal. However, it remains unclear what role TSS plays in the pathogenesis of IIH, its visual prognosis, and response to treatment. Over the past decade, endovascular stenting of TSS has been proposed as a possible treatment for refractory $\mathrm{IIH} .{ }^{8,9}$ Some clinicians believe that the presence of obvious TSS should alter the management of patients with IIH, whereas others do not take the presence or degree of TSS into account when considering management strategies. ${ }^{9-11}$

Our aim was to determine the characteristics of TSS within an IIH cohort and to determine if the degree of anatomical TSS influences the clinical course or visual outcome of patients with IIH.

From the Departments of Ophthalmology (B.D.R., B.B.B., M.A.R., L.P.K., N.J.N., V.B.), Neurology (B.B.B., N.J.N., V.B.), Neurological Surgery (N.J.N.), and Radiology and Imaging Sciences (A.M.S.), Emory University School of Medicine; and Rollins School of Public Health and Laney Graduate School (B.B.B.), Emory University, Atlanta, GA.

Go to Neurology.org for full disclosures. Funding information and disclosures deemed relevant by the authors, if any, are provided at the end of the article. 
METHODS Standard protocol approvals, registrations, and patient consents. This retrospective study was granted a waiver of informed consent by our institutional review board.

Study conduct. Patients with definite IIH according to the most recent diagnostic criteria ${ }^{12}$ were identified using our database, and their medical records were reviewed to determine eligibility. Patients were included if they had a high-quality contrast-enhanced magnetic resonance venography (MRV) study performed at our institution since September 2009, when an improved technology for MRV was implemented. Patients were excluded if the MRV was deemed to be of inadequate quality by our neuroradiologist (A.M.S.). All included patients were evaluated in a standardized fashion by experienced neuro-ophthalmologists (B.B.B., V.B., N.J.N.), including documentation of body habitus, detailed neuroophthalmic examination with formal visual field (VF) testing (static perimetry using a Humphrey automated perimeter $[\mathrm{HVF}]$ or kinetic perimetry using a Goldmann perimeter [GVF]), review of lumbar puncture (LP) data (including CSF opening pressure), and review of neuroimaging tests. Management strategies were similar for all patients included in this study, including diagnostic LP, discontinuation of any medications possibly associated with the development of raised ICP, the use of acetazolamide with total daily dosages between 500 and $1,500 \mathrm{mg}$, and recommendation for weight loss. Surgery (either CSF-shunting procedure or optic nerve sheath fenestration) was performed in fulminant forms,${ }^{13}$ in patients with severe visual loss at presentation, when the visual function was abnormal at baseline and did not improve within a few weeks, when visual function deteriorated despite maximal medical therapy, or for intractable headaches clearly related to elevated ICP. None of our patients underwent endovascular venous stenting

In order to compare HVF and GVF tests, we used a 3-point scale to evaluate the severity of VF loss in our patient population. The degree of final VF loss was graded on a 0 to 2 point scale: 0 )

\section{Figure 1 Reformatting and measurement of the degree of transverse sinus} stenosis
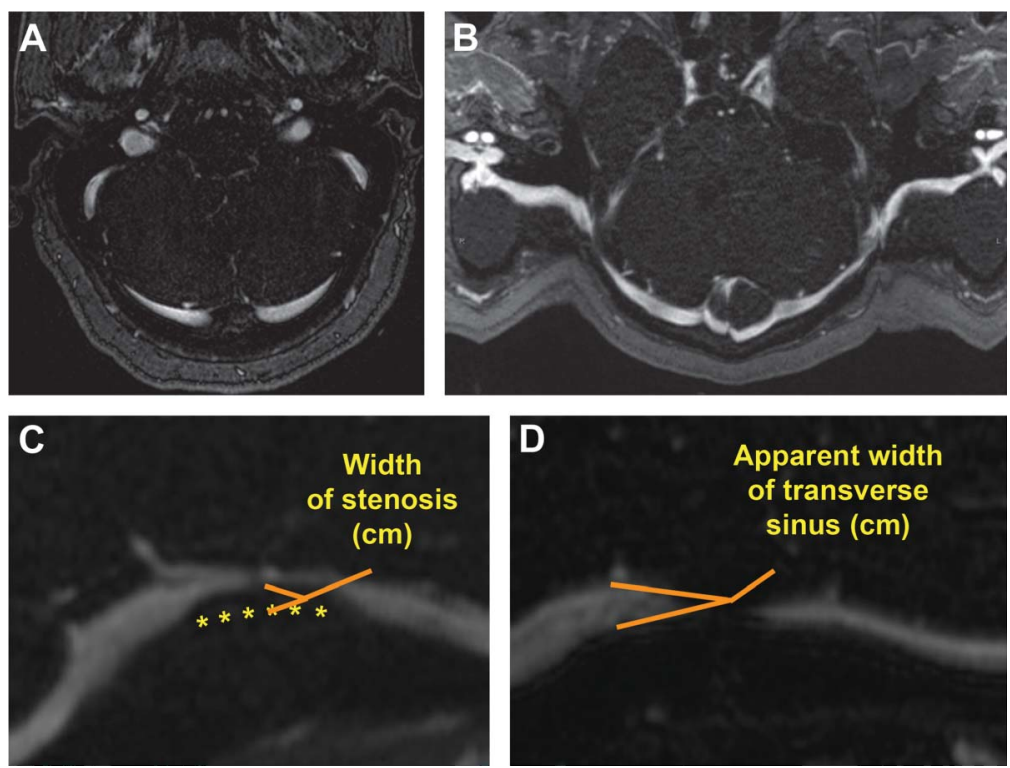

The entire course of the transverse sinus in a single axial magnetic resonance venography image (A) is difficult to visualize, but with curved reformatting $(B)$, the entire course of the transverse sinus is apparent. The percent stenosis was calculated by dividing the width of stenosis (C) by the width of the adjacent normal-appearing transverse sinus (D), yielding the percent stenosis. normal or enlarged blind spot, 1) mild or moderate VF deficit, 2) severe VF deficit by consensus review of 2 neuro-ophthalmologists (B.D.R. and B.B.B.). HVF mean deviations were recorded when available.

Because surgical procedures are rarely needed in the management of IIH and usually reflect a more severe form of IIH (despite an often good outcome if the procedure was performed at an appropriate time), we chose to describe the overall clinical course in addition to the final VF outcome. Charts of eligible patients were reviewed to determine the clinical course. We classified all eligible patients into 2 groups: those with "good" and those with "poor" clinical courses based on review of the entire clinical course. The group considered to have a poor clinical course included patients who had fulminant disease, ${ }^{13}$ had progressive VF defects, or required a surgical procedure for IIH management. Patients with a good clinical course had none of the poor clinical features.

MRI was performed using a standardized protocol at either 3.0 T (Siemens Trio, Erlangen, Germany) or $1.5 \mathrm{~T}$ (Siemens Avanto or GE Signa, Milwaukee, WI) using a standard head coil. All patients underwent standardized contrast-enhanced MRI along with contrast-enhanced MRV. The MRI/MRV protocol included routine precontrast axial diffusion-weighted, T1-weighted, T2-weighted gradient-recalled echo (GRE) and sagittal T1-weighted images. An axial precontrast MRV mask was obtained (repetition time 4-6 msec, echo time 1-2 msec, flip angle of 22-30 degrees with slice thickness of 0.8-1.4 mm). A standard dose $(0.1 \mathrm{mmol} / \mathrm{kg})$ IV gadolinium-based contrast agent (Multihance, Bracco Diagnostics Inc.) was administered at $2.0 \mathrm{cc} / \mathrm{s}$, and the axial MRV sequence was repeated $60 \mathrm{sec}-$ onds following contrast administration. Postcontrast axial T2weighted and T1-weighted and sagittal volumetric T1-weighted GRE images of the brain were then acquired. The precontrast MRV dataset was subtracted from the postcontrast dataset, and multiple oblique maximum intensity projections (MIP) were generated from this subtracted dataset with rotation around the craniocaudal axis ("spin") or the transverse axis ("nod") at 6-degree increments.

Curved reformats of each transverse sinus (TS) (figure 1) were generated using the source images for each patient. The area of greatest stenosis of each TS was identified and measured. Appropriately accounting for the workstation field of view, the length and width of the stenosis and the distance from the torcular to the midpoint of the stenosis were measured. The apparent width of the TS at the point of the greatest stenosis was measured based on the adjacent nonstenosed sinus. The percent of stenosis was calculated by dividing the width of the stenosis by the apparent normal width of the sinus. The average of the right and left TSs of each patient was calculated to provide a single measure of average percent stenosis for each patient. An average distance from the torcular was calculated in a similar fashion. Any sinus without a discernable stenosis was excluded from the calculation of the average distance from the torcular. Any sinus with multiple areas of stenosis was measured at the point of greatest stenosis. To incorporate the length of stenosis, a lengthweighted percent stenosis was calculated by a weighted average of the 2 sides' percent stenosis by the respective length of stenosis. To provide an estimate of total venous outflow capacity, a residual area was measured as the sum of the squares of the difference of sinus width and stenosis width bilaterally. All MRV reformatting and measurements were performed by a single investigator (B.D.R.) blinded to the clinical outcome and the measurements were confirmed to be accurate by a neuroradiologist (A.M.S.). Asymmetry between the 2 TSs was noted and right or left TS dominance was documented. Codominance was defined as less 
than $5 \%$ difference between the 2 sinuses. Hypoplastic or aplastic TSs were also recorded.

Statistical analysis. Statistical analysis was performed with R: A language and environment for statistical computing ( $R$ Foundation for Statistical Computing, http://www.R-project.org). Medians and interquartile ranges (IQR) were reported for continuous data; percentages were reported for categorical data. Distributions of continuous variables by categorical variables were compared using the Wilcoxon Mann-Whitney rank sum or Kruskal-Wallis tests depending on the number of subgroups. Proportions were compared using Fisher exact tests. Linear regression analysis was performed between continuous variables. Two-tailed $p$ values $<0.05$ were considered statistically significant.

RESULTS Seventy-one patients with definite IIH had a contrast-enhanced MRI/MRV at our institution during the study enrollment period and were eligible for inclusion. Twenty patients were excluded due to either nonavailability of the data or a poor contrast bolus, leading to inadequate imaging for accurate stenosis measurement.

The 51 included IIH patients were mostly women (92\%), with median age 29 years and median body mass index $39 \mathrm{~kg} / \mathrm{m}^{2}$. Black patients constituted 53\% of our cohort, consistent with the patient population seen at our institution. All patients had a LP showing an elevated ICP, and the median CSF opening pressure was $35 \mathrm{~cm} \mathrm{H}_{2} \mathrm{O}$ (IQR 29-43 cm H $\mathrm{H}_{2} \mathrm{O}$ ). Three patients had LPs performed prior to MRV, but all of them had persistent bilateral TSS on MRV. Forty-three patients

\section{Figure 2 Distribution of the average percent stenosis}

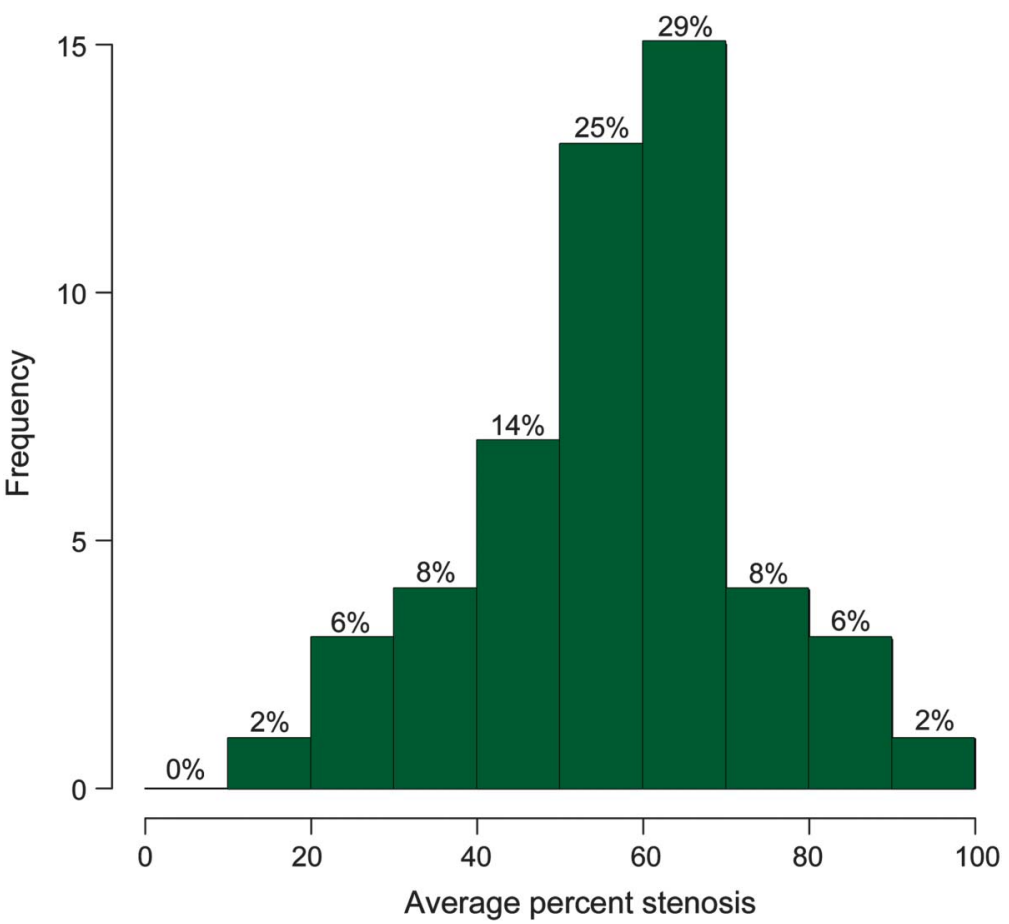

The average percent stenosis ranged from $19 \%$ to $96 \%$ with a median of $56 \%$ (interquartile range $49 \%-65 \%$ ). Seventy-one percent of patients had $50 \%$ or greater average percent stenosis. had good clinical courses, with 35 of these having no persistent VF loss. Eight patients (16\%) had clinical courses classified as poor. Two of these patients had no VF loss (VF score of 0 ), but had poor clinical courses due to intractable headache secondary to raised ICP relieved only by ventriculoperitoneal shunting.

By our methodology, all 51 patients had some degree of unilateral or bilateral TSS. Twenty-four patients (47\%) were right TS dominant, 19 (37\%) were left TS dominant, and $8(16 \%)$ were codominant. Five patients $(9.8 \%)$ had unilateral TSS, but 4 of these 5 patients $(80 \%)$ had a contralateral hypoplastic sinus. The range of the average percent stenosis was 19\%96\%; the median average percent stenosis of the distal TSS was 56\% (IQR 49\%-65\%) (figure 2), and the median average length weighted percent stenosis was $60 \%$ (IQR 51\%-70\%). Seventy-one percent of patients had $50 \%$ or greater average percent stenosis. The median residual area was $16.8 \mathrm{~mm}^{2}$ (range $0.49-67.3 \mathrm{~mm}^{2}$ ).

The degree of stenosis of the TS was not associated with the location of the stenosis along the TS, defined as the distance from the torcular to the midpoint of the stenosis $(-0.001 \mathrm{~cm}$ from torcular/percent stenosis, $p=0.84)$. The CSF opening pressure was not associated with either location along the TS $(+0.02 \mathrm{~cm}$ from torcular $/ \mathrm{cm} \mathrm{H}_{2} \mathrm{O}, p=0.11$ ), the degree of TSS (figure 3, $-0.0027 \%$ stenosis $\left./ \mathrm{cm} \mathrm{H}_{2} \mathrm{O}, p=0.28\right)$, or the residual area $\left(0.0311 \mathrm{~mm}^{2} / \mathrm{cm} \mathrm{H}_{2} \mathrm{O}, p=0.78\right)$. Of note, the patient with the highest opening pressure $(60 \mathrm{~cm})$ had the lowest average percent stenosis (19\%).

Comparing those patients with good clinical courses vs those with poor courses (figure 4), there was no difference in the average percent stenosis ( $62 \%$ vs $56 \%, p=0.44)$, the average length weighted percent stenosis $(64 \%$ vs $57 \%, p=0.23)$, or the residual area (17 vs $14, p=0.27$ ). The final VF deficit (figure 5) was not associated with either the average percent stenosis (VF grade 0: $58.1 \%$, VF grade 1: $54.0 \%$, VF grade $2: 57.6 \% ; p=0.38$ ) or the residual area (VF grade 0: $17 \mathrm{~mm}^{2}$, VF grade 1: $15 \mathrm{~mm}^{2}$, VF grade 2: $\left.18 \mathrm{~mm}^{2} ; p=0.81\right)$. Likewise, for the 47 patients who had HVFs, there was no correlation between the mean deviation (average of both eyes or worst eye) and either the average percent stenosis or the residual area. Having unilateral TSS did not correlate with the clinical course $(p=0.58)$ nor with the presence or absence of a VF defect $(p=1.0)$. However, none of the 5 patients with unilateral TSS had a poor clinical course and only 1 had some nasal VF loss at final evaluation.

There was no association between the location of the stenosis along the course of the TS and the clinical course (5.8 vs 6.2, $p=0.61$ ) or VF loss (VF grade 0 : 5.8, VF grade 1: 5.9, VF grade 2: 6.5; $p=0.58$ ). There were trends toward higher CSF opening 
Figure $3 \quad$ Linear regression of average percent transverse sinus stenosis by CSF opening pressure

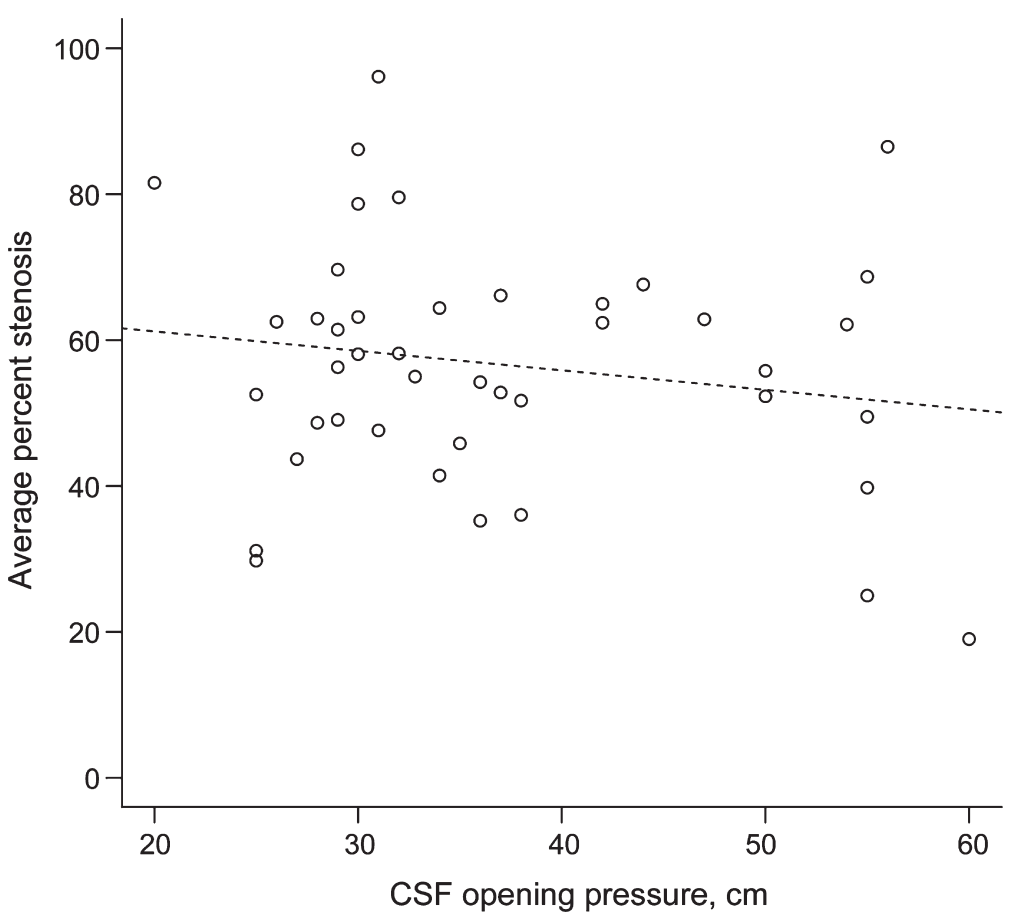

There was no relationship between the two (slope: -0.0027 percent stenosis $/ \mathrm{cm} \mathrm{H}_{2} \mathrm{O}, \mathrm{p}=0.28$ ).

pressure predicting both a poor clinical course (odds 1.9 times higher per $10 \mathrm{~cm}$ increase in CSF opening pressure, 95\% confidence interval 0.9-4.0, $p=0.10$ ) and greater VF loss (odds of one level increase in VF degree 1.8 times per $10 \mathrm{~cm}$ increase in CSF opening pressure, 95\% confidence interval 1.0-3.3, $p=0.054$ ).

DISCUSSION All of our patients with IIH had some degree of TSS, but we found no association between the degree of TSS in these patients and their clinical course, including the severity of their VF loss. While all of our patients with unilateral TSS had a good clinical course, and all but one had a normal VF grade, this finding did not reach significance. Even if patients with lower degrees of stenosis tend to have better outcomes, our study shows that higher degrees of stenosis do not necessarily portend a poor outcome. Indeed, the patient with the most severe stenosis had a good clinical course and normal VFs. This was surprising given the prevailing assumption that TSS is on a pathologic feedback loop that would be expected to increase ICP and thus lead to poorer outcomes. ${ }^{3,14}$

Prior studies have attempted to describe the stenoses seen in patients with IIH, in some cases semiquantitatively. ${ }^{15-18}$ Varying frequencies of TSS in patients with IIH have been reported, with generally a very high prevalence (65\%-100\%). ${ }^{15-17,19}$ These differences can be explained by differences in the imaging techniques used to observe the stenoses (i.e., digital subtraction angiography, time-of-flight MRV, measurement of pressure gradients). Prior to the development of more recent MRV techniques, interpretation of MRVs and quantification of TSS was plagued by "flow-gaps" that are likely artifactual in nature, related to turbulent flow. ${ }^{20}$ With recent advances in MRV technology and gadolinium-enhanced studies, these artifacts are minimized and TSS can be better defined. ${ }^{21}$

Another major difficulty in determining the true prevalence of TSS stems from the varying methodologies used in assessing stenosis. Because there is no accepted definition of what constitutes clinically relevant venous stenosis, ${ }^{22,23}$ we used direct measurements of the stenoses rather than determining only the presence or absence of stenosis or broadly

Figure 4 Distribution of the average percent stenosis and residual area by clinical course
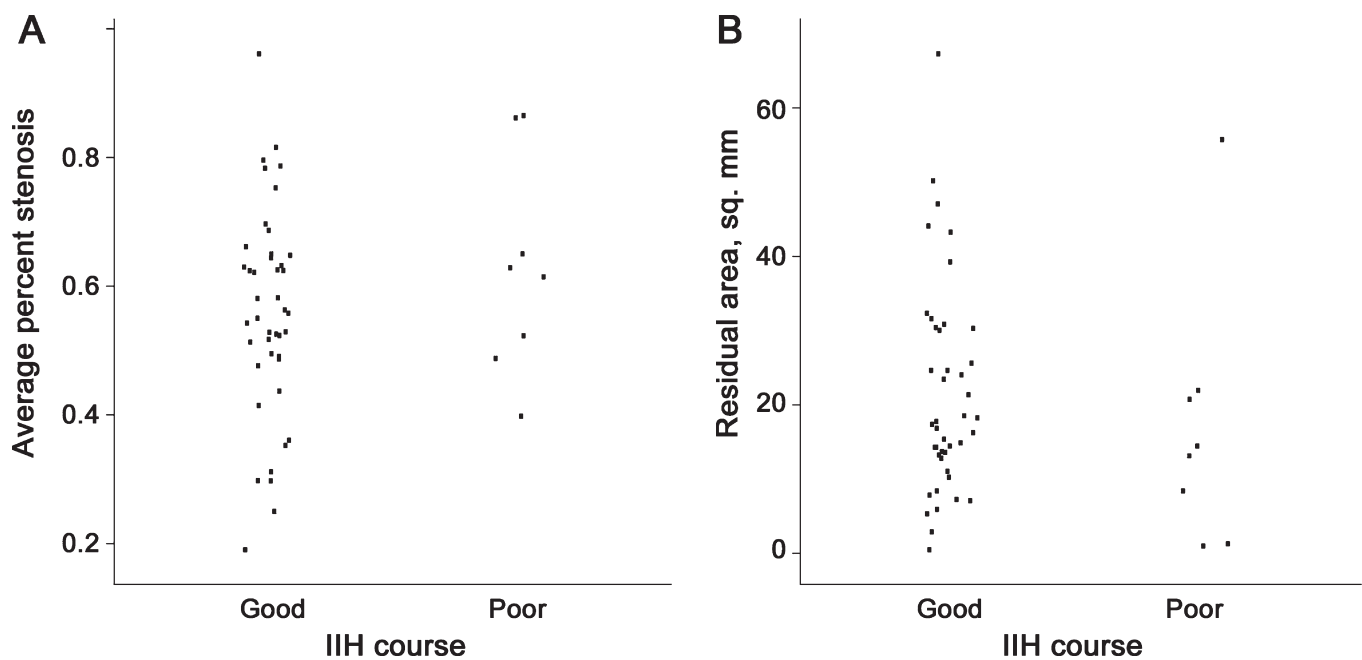

Average percent stenosis (A) and residual area (B). There was no association with either transverse sinus stenosis $(p=0.44)$ or residual area $(p=0.27)$. $I \mathrm{H}=$ idiopathic intracranial hypertension. 

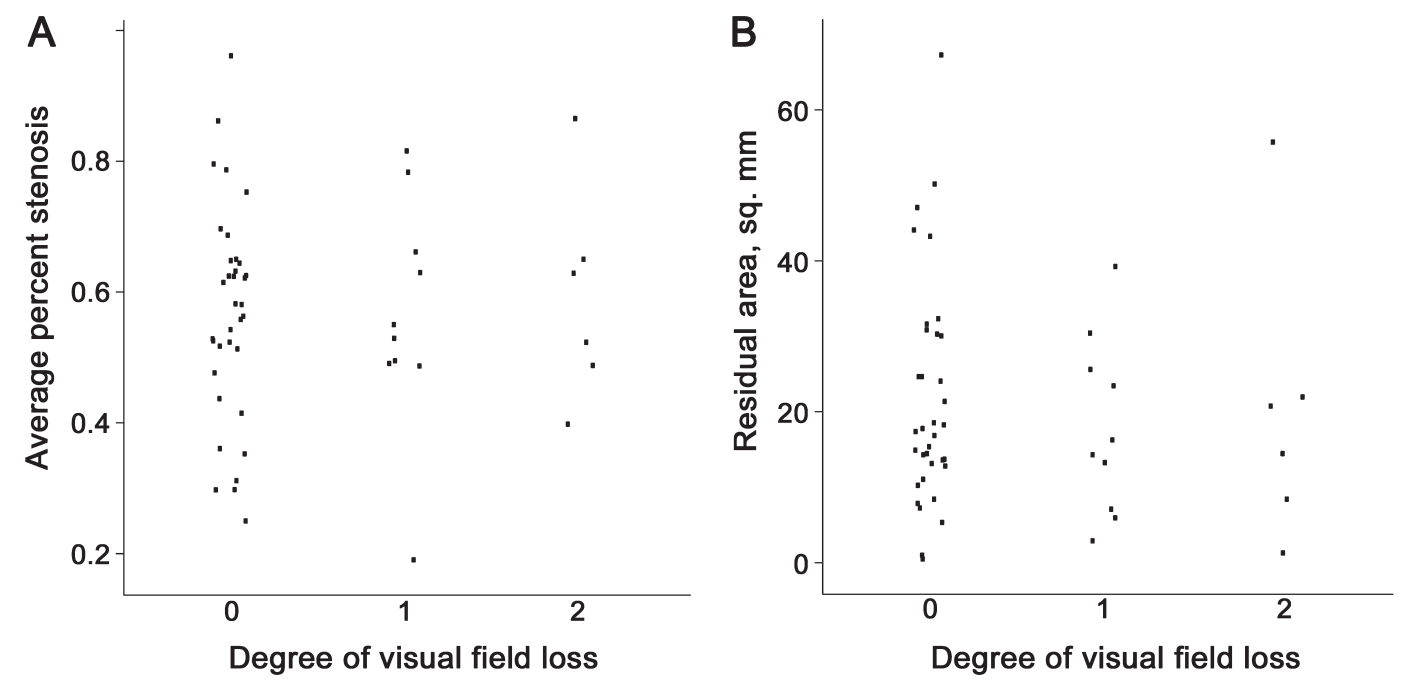

Average percent stenosis (A) and residual area (B). Degree of visual field loss: $0=$ none, $1=\mathrm{mild} /$ moderate, $2=$ severe. There was no association with either transverse sinus stenosis $(p=0.98)$ or residual area $(p=0.81)$.

categorizing the degree of stenosis. From our absolute measurements, we derived 2 quantitative global measures of venous stenosis: average percent stenosis and residual area. If stenosis is defined as any narrowing of the TS compared to adjacent "normal" TS, we found that stenosis occurs almost universally in IIH.

In a previous study of TSS, 3 neuroradiologists blinded to the diagnosis of $\mathrm{IIH}$ reviewed contrastenhanced images acquired using the auto-triggered elliptic-centric-ordered (ATECO) gadolinium-enhanced MRV technique. ${ }^{21}$ They devised a semiquantitative scale to measure the degree of TSS. Based on a cutoff that they derived by comparison to controls, TSS was present in over $90 \%$ of patients with IIH but in only $7 \%$ of controls, leading them to conclude that TSS is an expected finding in IIH. This concurs with our findings, although we did not include control patients to address the prevalence of TSS in comparison to normal patients. Our MRI technique is similar to the ATECO technique used in their study, ${ }^{17}$ but we believe our technique has several advantages over the ATECO technique, which is proprietary to the other investigators' institution. Our technique has a shorter acquisition time ( 3 minutes vs 4.5 minutes), requires minimal postprocessing, uses a predetermined time delay rather than being triggered when contrast reaches a given location (resulting in no mistimed studies in our cases), and is performed using commercially available technology.

In agreement with prior investigators, ${ }^{17}$ we found no association between CSF opening pressure and the degree of TSS. This lack of association may be explained by the development of collateral flow, thereby maintaining overall venous resistance. Blood flow through enlarged dural veins in superior sagittal sinus occlusion has been demonstrated, and may represent one collateral path. ${ }^{24}$ Two additional paths include the vein of Labbe $^{25}$ (a cortical vein that drains the lateral surface of the hemispheres) and the occipital sinus ${ }^{26}$ (draining directly from the torcular). Both of these structures can, albeit with variation, drain into the sigmoid sinus, bypassing the distal TS where the majority of stenoses in patients with IIH occur.

Given recent literature ${ }^{27-30}$ reporting improvement in papilledema, headache, and CSF opening pressure with endovascular stenting of TSS, this procedure has become a potential treatment strategy for IIH. Some investigators have suggested performing systematic catheter venography with measurement of pressure gradients to determine if the stenosis is hemodynamically significant; if so, stenting is recommended. ${ }^{27}$ Our study suggests that more severe degrees of anatomic TSS are not a reliable indicator of poor prognosis in IIH, perhaps because the effects of these gradients are mitigated by collateral flow. Thus, we believe that presence and severity of anatomic TSS should not be used alone to guide the aggressiveness of treatment and should not necessarily prompt invasive venography for manometry and pressure gradient measurement. However, if further studies demonstrate long-term safety of TS stenting, this procedure may prove to be a reasonable option for patients with IIH in whom the clinical course is poor and the usual therapeutic options are exhausted.

One limitation of our study is the lack of a comparison group, limiting our ability to assess the degree of stenosis typical of normal individuals. In addition, while our quantitative measurements have advantages over qualitative and semiquantitative measures, our technique only assesses the point of maximal stenosis instead of the 
overall contour of the venous sinus or magnetic resonance flow-related changes, either of which could provide additional information regarding the severity of stenosis. However, the simplicity and noninvasive nature of our techniques for measuring the anatomic TSS will allow for more widespread application in clinical practice. Finally, currently available noninvasive techniques do not allow the easy and reliable measurement of the venous pressure gradient across a TSS. Thus, it remains possible that pressure gradient measurements across a TSS could predict the clinical course or outcome of patients with IIH. While we are unable to make any conclusions based on our data regarding the relationship between venous pressures and IIH, it is likely that the anatomic presence of TSS on MRV is not helpful for making treatment decisions for patients with IIH.

We present the only study, to our knowledge, which examines the relationship between clinical course and TSS among patients with IIH. Our quantitative measures of the TSs were not predictive of the clinical course of patients with IIH, suggesting that other factors such as those previously associated with poor outcomes in $\mathrm{IIH}^{14,31,32}$ (i.e., black race, male sex, anemia, recent weight gain, and morbid obesity) are more relevant than the anatomic degree of TSS. Most importantly, our study does not support the notion that patients with IIH with high-grade stenosis on MRV require TS stenting or need different management than patients with IIH without severe TSS in order to prevent poor outcomes.

\section{AUTHOR CONTRIBUTIONS}

Bryan Riggeal, MD: study design, acquisition/analysis/interpretation of data, drafting/revising manuscript for content. Beau Bruce, MD, MS: study design, analysis/interpretation of data, revising manuscript for content. Amit Saindane, MD: study design, interpretation of data, revising manuscript for content. Maysa Ridha, MD: interpretation of data. Linda Kelly, MD: interpretation of data. Nancy J. Newman, MD: study design, interpretation of data, revising manuscript for content. Valérie Biousse, MD: study design, interpretation of data, revising manuscript for content.

\section{STUDY FUNDING}

Supported in part by an unrestricted departmental grant (Department of Ophthalmology) from Research to Prevent Blindness, Inc., New York, NY, and by NIH/NEI core grant P30-EY06360 (Department of Ophthalmology).

\section{DISCLOSURE}

B. Riggeal reports no disclosures. B. Bruce received research support from Teva Pharmaceuticals via an Emory University-approved research agreement; received the American Academy of Neurology Practice Research Fellowship; and receives research support from the NIH (K23-EY019341) and from Pfizer via an Emory University-approved research agreement. A. Saindane, M. Ridha, and L. Kelly report no disclosures. N. Newman received the Research to Prevent Blindness and a Lew R. Wasserman Merit Award. V. Biousse received research support from the NIH (PHS Grant UL1-RR025008). Go to Neurology.org for full disclosures.

Received May 24, 2012. Accepted in final form September 7, 2012.

\section{REFERENCES}

1. Corbett JJ, Savino PJ, Thompson HS, et al. Visual loss in pseudotumor cerebri: follow-up of 57 patients from five to 41 years and a profile of 14 patients with permanent severe visual loss. Arch Neurol 1982;39:461-474.

2. Binder DK, Horton JC, Lawton MT, McDermott MW. Idiopathic intracranial hypertension. Neurosurgery 2004; 54:538-551.

3. Karahalios DG, Rekate HL, Khayata MH, Apostolides PJ. Elevated intracranial venous pressure as a universal mechanism in pseudotumor cerebri of varying etiologies. Neurology 1996;46:198-202.

4. Bateman GA, Stevens SA, Stimpson J. A mathematical model of idiopathic intracranial hypertension incorporating increased arterial inflow and variable venous outflow collapsibility. J Neurosurg 2009;110:446-456.

5. Rohr A, Dorner L, Stingele R, Buhl R, Alfke K, Jansen O. Reversibility of venous sinus obstruction in idiopathic intracranial hypertension. AJNR Am J Neuroradiol 2007;28: 656-659.

6. De Simone R, Marano E, Fiorillo C, et al. Sudden re-opening of collapsed transverse sinuses and longstanding clinical remission after a single lumbar puncture in a case of idiopathic intracranial hypertension: pathogenetic implications. Neurol Sci 2005;25:342-344.

7. Lee SW, Gates P, Morris P, Whan A, Riddington L. Idiopathic intracranial hypertension; immediate resolution of venous sinus "obstruction" after reducing cerebrospinal fluid pressure to $<10 \mathrm{~cm} \mathrm{H}_{2} \mathrm{O}$. J Clin Neurosci 2009; 16:1690-1692.

8. Ahmed RM, Wilkinson M, Parker GD, et al. Transverse sinus stenting for idiopathic intracranial hypertension: a review of 52 patients and of model predictions. AJNR Am J Neuroradiol 2011;32:1408-1414.

9. Ahmed R, Friedman DI, Halmagyi GM. Stenting of the transverse sinuses in idiopathic intracranial hypertension. J Neuroophthalmol 2011;31:374-380.

10. Bruce BB, Biousse V, Newman NJ. Update on idiopathic intracranial hypertension. Am J Ophthalmol 2011;152: 163-169.

11. Wall M. Idiopathic intracranial hypertension. Neurol Clin 2010;28:593-617.

12. Friedman DI, Jacobson DM. Diagnostic criteria for idiopathic intracranial hypertension. Neurology 2002;59:1492-1495.

13. Thambisetty M, Lavin PJ, Newman NJ, Biousse V. Fulminant idiopathic intracranial hypertension. Neurology 2007;68:229-232.

14. Bruce BB, Preechawat P, Newman NJ, Lynn MJ, Biousse V. Racial differences in idiopathic intracranial hypertension. Neurology 2008;70:861-867.

15. Higgins JN, Gillard JH, Owler BK, Harkness K, Pickard JD. MR venography in idiopathic intracranial hypertension: unappreciated and misunderstood. J Neurol Neurosurg Psychiatry 2004;75:621-625.

16. King JO, Mitchell PJ, Thomson KR, Tress BM. Manometry combined with cervical puncture in idiopathic intracranial hypertension. Neurology 2002;58:26-30.

17. Farb RI, Vanek I, Scott JN, et al. Idiopathic intracranial hypertension: the prevalence and morphology of sinovenous stenosis. Neurology 2003;60:1418-1424.

18. Johnston I, Kollar C, Dunkley S, Assaad N, Parker G. Cranial venous outflow obstruction in the pseudotumour syndrome: incidence, nature and relevance. J Clin Neurosci 2002;9:273-278. 
19. Fera F, Bono F, Messina D, et al. Comparison of different MR venography techniques for detecting transverse sinus stenosis in idiopathic intracranial hypertension. J Neurol 2005;252:1021-1025.

20. Ayanzen RH, Bird CR, Keller PJ, McCully FJ, Theobald MR, Heiserman JE. Cerebral MR venography: normal anatomy and potential diagnostic pitfalls. AJNR Am J Neuroradiol 2000;21:74-78.

21. Farb RI, Scott JN, Willinsky RA, Montanera WJ, Wright GA, terBrugge KG. Intracranial venous system: gadolinium-enhanced three-dimensional MR venography with auto-triggered elliptic centric-ordered sequence: initial experience. Radiology 2003;226:203-209.

22. Bergan JJ, ed. The Vein Book. Burlington, MA: Elsevier Academic Press; 2007:545.

23. Mullan CP, Siewert B, Kane RA, Sheiman RG. Can Doppler sonography discern between hemodynamically significant and insignificant portal vein stenosis after adult liver transplantation? AJR Am J Roentgenol 2010;195:1438-1443.

24. Ivanov A, Komkov D, Oliushin VE, Guliaev DA, Sebelev KI. Alternative ways of collateral venous flow in occlusion of superior sagittal sinus [in Russian]. Zh Vopr Neirokhir Im N N Burdenko 2010;4:10-14.

25. Kanno T, Kasama A, Suzuki H. Safety of ablation of the sigmoid and transverse sinuses: an experimental study. Skull Base Surg 1993;3:146-151.
26. Kobayashi K, Suzuki M, Ueda F, Matsui O. Anatomical study of the occipital sinus using contrast-enhanced magnetic resonance venography. Neuroradiology 2006; 48:373-379.

27. Kumpe DA, Bennett JL, Seinfeld J, Pelak VS, Chawla A, Tierney M. Dural sinus stent placement for idiopathic intracranial hypertension. J Neurosurg 2012;116:538548.

28. Paquet C, Poupardin M, Boissonnot M, Neau JP, Drouineau J. Efficacy of unilateral stenting in idiopathic intracranial hypertension with bilateral venous sinus stenosis: a case report. Eur Neurol 2008;60:47-48.

29. Higgins JN, Cousins C, Owler BK, Sarkies N, Pickard JD. Idiopathic intracranial hypertension: 12 cases treated by venous sinus stenting. J Neurol Neurosurg Psychiatry 2003;74:1662-1666.

30. Donnet A, Metellus P, Levrier O, et al. Endovascular treatment of idiopathic intracranial hypertension: clinical and radiologic outcome of 10 consecutive patients. Neurology 2008;70:641-647.

31. Orcutt JC, Page NG, Sanders MD. Factors affecting visual loss in benign intracranial hypertension. Ophthalmology 1984;91:1303-1312.

32. Biousse V, Rucker JC, Vignal C, Crassard I, Katz BJ, Newman NJ. Anemia and papilledema. Am J Ophthalmol 2003;135:437-446.

\section{Save These Dates for AAN CME Opportunities!}

Mark these dates on your calendar for exciting continuing education opportunities, where you can catch up on the latest neurology information.

\section{AAN Annual Meeting}

- March 16-23, 2013, San Diego, California, San Diego Convention Center

\section{Guide the Future of Neurology_Become a Mentor!}

The Academy's Neurology Career Center is working to bring experienced members together with members who seek guidance on their career path. AAN Mentor Connect needs volunteer Mentors who are willing to share their expertise, insights, and experiences with Mentees.

This flexible program, available only to AAN members, matches prospective Mentors and Mentees, and enables you to develop a plan with the Mentee that has a mutually agreeable schedule and expectations.

Enjoy the personal satisfaction of making a valued contribution to the career of a fellow AAN member. Visit www.ann.com/view/Mentor to learn more and register to be a Mentor today. 